(2) Open Access Full Text Article

\title{
Features of magnetic resonance imaging brain in eclampsia: clinicoradiologic correlation
}

\section{Fatima Mubarak \\ Muhammad Idris \\ Quratulain Hadi}

Department of Radiology, Aga Khan University Hospital, Karachi, Pakistan

This article was published in the following Dove Press journal:

Reports in Medical Imaging

8 August 2012

Number of times this article has been viewed

Objective: Eclampsia is a gestational hypertensive condition that typically occurs after 20 weeks of pregnancy and is characterized by hypertension, peripheral edema, proteinuria, and seizures. Magnetic resonance imaging (MRI) plays a vital role in the diagnosis and management of these patients, so it is essential to describe features of the brain MRI in these cases.

Methods: MRI was performed on eleven consecutive patients with eclampsia. All patients underwent follow-up neurologic examinations until all symptoms resolved. Nine of those eleven patients underwent follow-up MRI. The clinical signs and symptoms were correlated with findings on initial and follow-up MRI.

Results: MRI typically demonstrated bilateral hyperintense lesions on T2-weighted images and hypointense lesions on T1-weighted images without diffusion restriction. MRI abnormalities are most commonly located in the distribution of the posterior cerebral circulation mainly in occipital and parietal lobes, and are associated with visual disturbances and dizziness. Almost all lesions seen at MRI in patients with eclampsia were reversible in our series of patients.

Conclusion: Involvement of the parietal and occipital lobes is common in patients with eclampsia, and the signal abnormalities on MRI are reversible if recognized and treated early.

Keywords: pregnancy, seizures, hypertension, brain, MRI findings, reversible

\section{Introduction}

Eclampsia is a gestational condition that typically occurs after 20 weeks of pregnancy and is characterized by hypertension, peripheral edema, proteinuria, and seizures. ${ }^{1}$ If not diagnosed and treated early it may be associated with multiple neurologic complications, including focal neurologic deficits, altered mental status, and coma. ${ }^{2}$ The neurologic manifestations of eclampsia are very similar to hypertensive encephalopathy, and features on magnetic resonance imaging (MRI) in such patients are also identical and show edema in subcortical white matter and cortex, which predominantly involve the occipital lobes. ${ }^{3}$ These signs and symptoms are usually resolved completely at delivery of the child and placenta but may take up to 6 weeks. Fatalities are unusual, but if seen these may be due to cerebral hemorrhage, which occurs in patients with thrombocytopenia. ${ }^{4}$ This may occur in isolation or as a part of HELLP (hemolysis, elevated liver enzymes, low platelets) syndrome. ${ }^{5}$ The manifestations are due to the disturbance of autoregulation of the cerebral vasculature as a result of acutely raised systemic blood pressure. It is believed to be most commonly caused by vasogenic edema as a result of the breakthrough of autoregulation, which causes the escape of fluid from the intravascular compartment into the interstitium. ${ }^{6}$

Correspondence: Fatima Mubarak Radiology Department, Aga Khan University Hospital, Stadium Road, PO Box 3500, Karachi, 74800, Pakistan Tel +92 2148592073

Email mubarakfatima@hotmail.com 
The purpose of our study was to evaluate the MR findings in eclampsia and to determine whether these findings can be of any value in determining the prognosis of this disorder. Our observation confirms that MRI typically demonstrates bilateral hyperintense lesions on T2-weighted images and hypointense lesions on T1-weighted images. These lesions represent typical radiologic involvement of the brain in eclampsia. However, there may be the presence of similar lesions in the brain stem, basal ganglia, deep white matter, and splenium of corpus callosum occasionally.

\section{Materials and methods}

This is a retrospective cross-sectional descriptive study conducted at Aga Khan University Hospital, Karachi, Pakistan, in the Department of Radiology in collaboration with the Department of Medicine. Eleven consecutive obstetric patients with $20+$ weeks' gestation and eclampsia were selected over a period of 2 years from January 2007 to January 2009 (Table 1). The study was conducted after approval from the research ethics committee of our university hospital. The inclusion criteria for the study were generalized tonic clonic seizures, high blood pressure $(>140 / 90 \mathrm{mmHg})$, and proteinuria. Informed written consent was taken from all to conduct MRI scanning. Initial MRI was performed within 24 hours of the onset of seizures. MRI brain was performed on a 1.5 Tesla (Seimens Avanto, Siemens Medical Solutions USA, Inc, Malvern, PA) MRI machine. The imaging protocols in all patients included axial and sagittal T2-weighted (TE 100/TR 3660), axial T1-weighted TE 10/TR 100, and coronal FLAIR and diffusion-weighted sequences.

In four patients, intravenous contrast demeglumine gadopentetate (Magnevist, Schering, Berlin, Germany) was given. In addition to MRI brain, computed tomography scan brain was also performed in four patients to rule out subarachnoid hemorrhage. The time interval for follow-up studies ranged from 2 weeks to 2 months. Follow-up was not performed in two patients; one had a normal initial study, and the other patient refused rescanning. The MRI was read by two radiologists independently. However, contradictory observations, if any, were resolved by consensus.

\section{Results}

Among our series of patients the age range was 18-36 years with a mean age of 26 years. Ten of the eleven patients were $\mathrm{P} 1+0$, and one was $\mathrm{P} 2+0$. All these patients were admitted through the emergency department of our hospital.

\section{Clinical findings}

All of these patients had fits and headaches, three had dizziness, two had disorientation and impaired vision, and one had focal neurologic deficits including dysarthria and ataxia. In one of the two patients with impaired vision, one progressed to cortical blindness. Fundoscopy in this patient showed papilloedema. All of these patients were hypertensive. The range of systolic blood pressure was 140-180 $\mathrm{mmHg}$ with an average of $161 \mathrm{mmHg}$, and the range of diastolic blood pressure was $75-120 \mathrm{mmHg}$ with average of $99 \mathrm{mmHg}$. Except for two patients, the signs and symptoms of these patients resolved with symptomatic treatment within 9 days.

\section{MRI findings}

Initial MRI was done in all patients within 24 hours of the onset of seizures. In $9 \%$ (one of eleven) of these patients the initial MRI was normal, while in $91 \%$ (ten of eleven) of these patients there were multiple findings on MRI, which includes areas of abnormal signals in the subcortical and deep white

Table I Magnetic resonance imaging (MRI): clinical correlation in patients with eclampsia

\begin{tabular}{|c|c|c|c|c|c|c|c|}
\hline $\begin{array}{l}\text { Patient } \\
\text { no }\end{array}$ & $\begin{array}{l}\text { Age } \\
\text { (y) }\end{array}$ & Parity & $\begin{array}{l}\text { Blood pressure } \\
(\mathrm{mmHg})\end{array}$ & $\begin{array}{l}\text { Neurologic } \\
\text { symptoms }\end{array}$ & $\begin{array}{l}\text { Location of lesions } \\
\text { at initial MRI }\end{array}$ & $\begin{array}{l}\text { Residual } \\
\text { neurologic deficits }\end{array}$ & $\begin{array}{l}\text { Follow-up } \\
\text { MRI findings }\end{array}$ \\
\hline I & 25 & $\mathrm{PI}+0$ & $160 / 110$ & Headache/fits & Occipital & No & Normal \\
\hline 2 & 24 & $\mathrm{PI}+0$ & $180 / 120$ & Headache/fits & Occipital & No & Normal \\
\hline 3 & 18 & $\mathrm{GIPO}+0$ & $149 / 75$ & Fits & Parietal & No & Normal \\
\hline 4 & 22 & $\mathrm{PI}+0$ & $150 / 80$ & Disorientation/fits & Parietal & Ataxia & Normal \\
\hline 5 & 32 & $\mathrm{PI}+0$ & $180 / 120$ & Vertigo/fits & Parietal & No & Normal \\
\hline 6 & 23 & $\mathrm{PI}+0$ & $172 / 100$ & Dizziness/fits & Parietal & No & Normal \\
\hline 7 & 29 & $\mathrm{PI}+0$ & $160 / 100$ & Impaired vision/fits & Occipital & Papillodema & Normal \\
\hline 8 & 36 & $\mathrm{G} 2 \mathrm{PI}+\mathrm{I}$ & $150 / 90$ & Impaired vision/fits & Occipital & No & Normal \\
\hline 9 & 25 & $\mathrm{GIPO}+0$ & $160 / 110$ & Headache/fits & Occipital & No & Normal \\
\hline 10 & 25 & $\mathrm{PI}+0$ & $180 / 90$ & Dizziness/fits & Parietal & No & Refused \\
\hline II & 32 & $\mathrm{GIPO}+0$ & $140 / 90$ & Disorientation/fits & Normal & No & Not performed \\
\hline
\end{tabular}

Abbreviations: $P$, No of pregnancy; G, gravida. 
matter mainly in the occipital and parietal regions. These lesions were hypointense on $\mathrm{T} 1 \mathrm{~W}$ images and hyperintense on $\mathrm{T} 2 \mathrm{~W}$ images with accentuation of the hyperintense signals on the FLAIR sequences. However, none of these lesions show diffusion restriction. In the patients in whom contrast was administered, no abnormal parenchymal or meningeal enhancement was seen. (Figure 1) None of the patients had abnormal signals in the brain stem or in the region of basal ganglia.

Follow-up MRI was done in nine of the eleven patients. Out of the two patients in which follow-up was not performed, one had an initial normal study, and the other refused a repeat scan as she was feeling claustrophobic and also her symptoms were resolved. The average duration of follow-up was 6 weeks to 2 months.

\section{Clinicoradiologic correlation}

It was noted that in patients with eclampsia and blood pressure more than $140 \mathrm{mmHg}$ systolic and $100 \mathrm{mmHg}$ diastolic and intractable seizures they developed abnormal signals in parietal and occipital lobes. As mentioned in previous studies too, ${ }^{7-13}$ these signals are suggestive of transient loss of arterial flow in the posterior circulation. Posterior circulation is

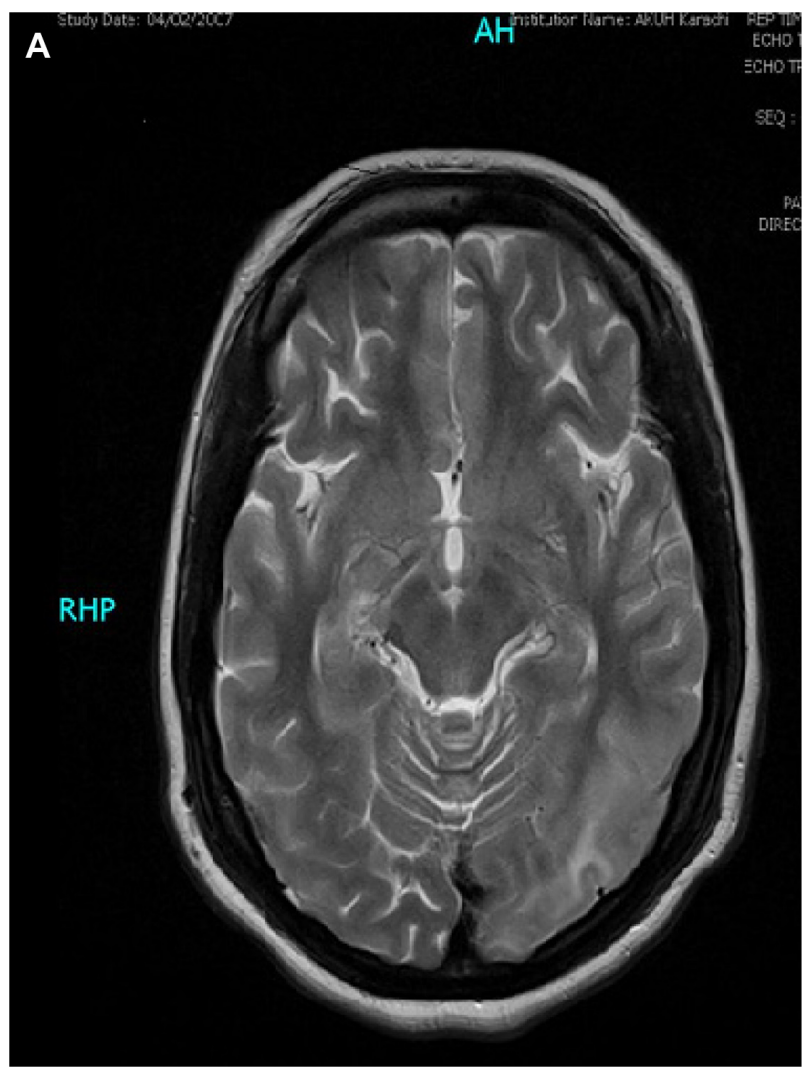

Figure I (Continued)
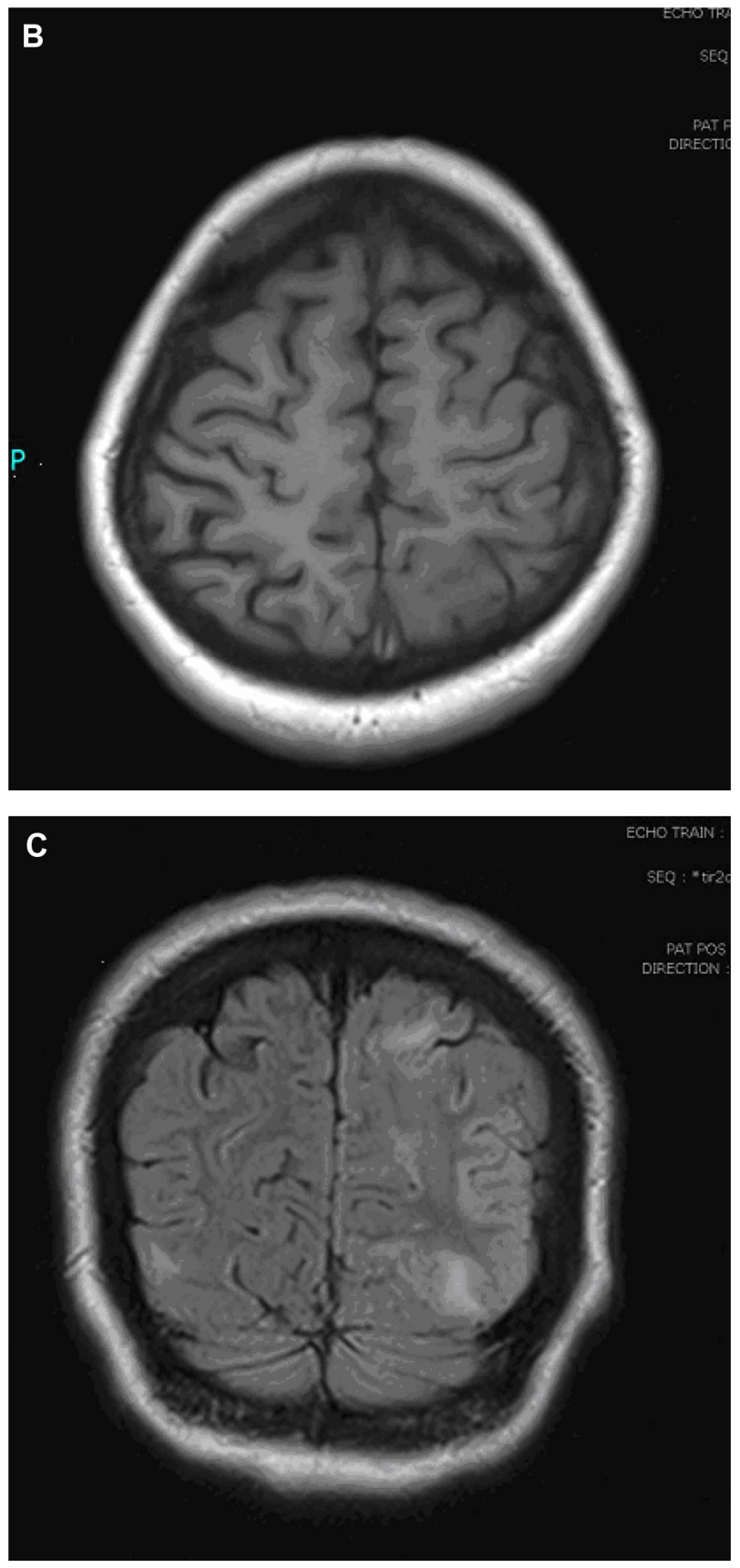

Figure I Abnormal signal intensity areas are noted in the left parieto-occipital regions associated with edema and effacement of ipsilateral cortical sulci. Minimal abnormal signals are also noted in the right posterior parietal region. (A) T2W axial magnetic resonance image (MRI). (B) TIW axial MRI. (C) Coronal FLAIR MRI. Abbreviations: MRI, magnetic resonance imaging.

mainly involved because of sparse autonomic innervations. However, with normalization of blood pressures, these patients usually show recovery in terms of their symptoms and imaging findings, as depicted in our results.

\section{Discussion}

Obstetric hemorrhages, eclampsia, and puerperal sepsis are commonly reported direct causes of maternal deaths in 
Pakistan as well as in other parts of the globe. It has been found that the presence of brain edema at MRI in patients who presented with pre-eclampsia-eclampsia and neurologic symptoms was associated with elevated blood pressure and hemodynamic changes in the posterior circulation. In some recent studies these findings are associated with altered red cell morphology and raised lactate dehydrogenase levels and indicate microangiopathic hemolysis, which suggests endothelial damage. Patients who had brain edema as compared with others had significantly increased uric acid and creatinine levels, which implied renal dysfunction, and increased hematocrit and white blood cell concentration, which likely reflected relative hemoconcentration owing to decreased intravascular volume. As mentioned in the findings of previous studies, investigators have shown that endothelial damage in patients with pre-eclampsia-eclampsia is not a result of hypertension but actually precedes substantial blood pressure increases. ${ }^{4}$ The endothelial dysfunction experienced by these patients is more likely related to circulating endothelial toxins or antibodies against the endothelium. The radiologic pattern observed in those patients was similar to that observed in patients who were not pregnant and who had severe hypertensive encephalopathy.

Although hypertensive encephalopathy can arise in patients with conditions in which there is acute systemic hypertension alone, it most commonly occurs in patients with conditions in which there is also pre-existing endothelial dysfunction or damage, such as systemic lupus erythematosus, cryoglobulinemia, or hemolytic uremic syndrome, and in patients undergoing cyclosporine and cisplatin therapy. Investigators in studies using single photon emission computed tomography and diffusion-weighted MRI have demonstrated that the brain edema in patients with hypertensive encephalopathy does not represent cytotoxic edema. Rather, the combination of acute hypertension and endothelial damage results in hydrostatic edema - a specific form of vasogenic edema characterized by the forced leakage of serum through capillary walls and into the brain interstitium - which, if severe enough, will be radiographically evident.

Cerebrovascular autoregulatory considerations may help to explain the pathogenesis and distribution of abnormalities in patients with hypertensive encephalopathy. Brain perfusion is maintained by an autoregulatory system of the small arteries and arterioles that have myogenic and neurogenic components. Endothelial damage may attenuate or abolish the myogenic response. The perivascular sympathetic nerves, which serve to protect the brain if the myogenic response is blunted or overwhelmed, travel in the adventitial layer of the cerebral vessels and are relatively protected from agents that cause endothelial damage. As the vertebrobasilar system and posterior cerebral arteries are sparsely innervated by sympathetic nerves, the occipital lobes and other posterior brain regions may be particularly susceptible to breakthrough of autoregulation with elevated systemic pressures. Investigators in recent Doppler ultrasonographic studies have demonstrated elevated cerebral perfusion pressures and reduced cerebrovascular resistance in patients with eclampsia, and increased regional cerebral blood flow to the occipital lobes has been documented in these patients who undergo single photon emission computed tomography and xenon computed tomography. Seizures were more frequent in patients with brain edema than in those with normal findings. This most likely reflects the irritative effects of fluid in the subcortical and cortical tissues. ${ }^{13,14}$ Some authors have suggested, on the basis of the common association of seizures with hypertensive encephalopathy, that the radiographic findings in patients with hypertensive encephalopathy actually may represent seizure edema. We have shown in this series and in previous studies, however, that radiographic abnormalities in patients with eclampsia can occur in the absence of seizures. In fact, as patients with hypertensive encephalopathy who do not experience seizures are less likely to come to neuroradiologic attention, they may be under-represented in this and other studies. Furthermore, brain edema is not frequently associated with idiopathic seizures, even in patients with status epilepticus; when it occurs, it tends to be unifocal, limited to the cortex, and have cytotoxic characteristics at diffusion-weighted imaging. ${ }^{15,16}$ The physical stresses of seizures may have contributed to the leukocytosis and hyperuricemia. ${ }^{17,18}$ Also, serum magnesium levels were significantly lower in the group with brain edema, as compared with the group with normal MRI findings.

Magnesium has been shown to be effective in reducing the occurrence of seizures in pre-eclampsia and may exert this effect by directly decreasing neuronal excitability, protecting the endothelium against damage by free radicals, or reducing cerebral perfusion pressures. However, more specific markers of endothelial dysfunction that have also been found to be released in patients with pre-eclampsia include fibronectin, tissue plasminogen activator, thrombomodulin, endothelin-1, and, in particular, von Willebrand factor. ${ }^{19}$ Measurement of these specific markers may be useful to evaluate endothelial integrity in patients who are pre-eclamptic, especially patients who are at risk for progression to hypertensive encephalopathy, such as those with severe headaches or 
other neurologic signs and symptoms. If this screening result is abnormal, treatment with appropriate antihypertensives may be initiated before hypertensive encephalopathy can develop. Recently, Zhou et $\mathrm{al}^{19}$ have reported the role of angiotensin receptor agonistic autoantibodies in the induction of pre-eclampsia in pregnant mice. However, its role in human subjects has not been established so far.

\section{Conclusion}

There is typical radiologic involvement of the brain in eclampsia, along with the occasional presence of lesions in the brain stem, basal ganglia, deep white matter, and splenium of corpus callosum. Awareness of these patterns and their clinical correlation is important when dealing with eclampsia.

\section{Disclosure}

The authors report no conflicts of interest in this work.

\section{References}

1. Khushk IA, Kazi RA. Maternal mortality: a priority in reproductive health. J Liaquat Uni Med Health Sci. 2006;5(2):48-49.

2. Uygar U, Mustafa G. Different clinical and imaging faces of posterior reversible leukoencephalopathy syndrome (PRES). Eur J Gen Med. 2010;7(2):230-233.

3. Lamy C, Oppenheim C, Meder JF, Mas JL. Neuroimaging in posterior reversible encephalopathy syndrome. $J$ Neuroimaging. 2004;14:89-96.

4. Annet M, Jan C, Jan G, Gerda G. Brain lesions several years after eclampsia. Am J Obstet Gynecol. 2009;200(5):5045.

5. Robert B, Rafael R, Carolina V. Brain apparent water diffusion coefficient magnetic resonance image during a prolonged visual aura. Headache. 2010;50(6):1045-1049.
6. Anderson K, Renato M. Ademar R. Hellp syndrome, reversible posterior leukoencephalopathy syndrome and eclampsia. Arq Neuropsiquiatr. 2009;67(4):1103-1105.

7. Bozek J,Zimmerman S, Rogove A. A pressing headache. Emergency Medicine News. 2010;32(2). doi:10.1097/01.EEM.0000368097.17205.74.

8. Steegers EA, von Dadelszen P, Duvekot JJ, Pijnenborg R. Pre-eclampsia. Lancet. 2010;376(9741):631-644.

9. Ritesh K, Rohith V, Shubha S, Vinay R. Late postpartum preeclampsia with posterior reversible encephalopathy syndrome. Indian J Med Sci. 2009;63(11):508-511.

10. Williams KP, Wilson S. Headache and seizure on postpartum day 5: late postpartum eclampsia. CMAJ. 2009;180(4):425-428.

11. Sandeep K, Ravindra R, Sudeep K. PRES (posterior reversible encephalopathy syndrome) and eclampsia. Review. The Internet Journal of Anesthesiology. 2009;22(1):23.

12. Jyotsana, Ashok K, Sunil B. Reversible blindness in severe preeclampsia and eclampsia. JK Science. 2004;6(1):43-45.

13. Fletcher JJ, Kramer AH, Bleck TP, Solenski NJ. Overlapping features of eclampsia and postpartum angiopathy. Neurocrit Care. 2009;11(2):199-209.

14. Murphy G, Martin N, Laila I. Management of Common Problems in Obstetrics and Gynecology. 5th ed. Oxford, UK: Blackwell Publishing Ltd; 2010.

15. Val EG, Bryan W. Headache and seizure on postpartum day 5: late postpartum eclampsia. CMAJ. 2009;180(4):425-428.

16. Aneesh BW, Taylor K, Pamela W. A 36-year-old woman with headache, hypertension, and seizure 2 weeks post partum. $N$ Engl $J$ Med. 2009;360:1126-1137.

17. Abdulrahman S, Sayed M, George K, Indulekha S. A rare cause of visual defect in a postpartum woman. Oman Med J. 2010;25(2):128-130.

18. Sahin AJ, Habib SS. A study of late postpartum eclampsia - 178 cases. Bangladesh Med J. 2008;37(2):52-55.

19. Zhou CC, Zhang Y, Irani RA, Zhang H, Mi T, Popek EJ. Angiotensin receptor agonistic autoantibodies induce pre-eclampsia in pregnant mice. Nat Med. 2008;14(8):855-862.
Reports in Medical Imaging

\section{Publish your work in this journal}

Reports in Medical Imaging is an international, peer-reviewed, open access journal publishing original research, reports, reviews and commentaries on all areas of medical imaging. The manuscript management system is completely online and includes a very quick and fair peer-review system, which is all easy to use.

\section{Dovepress}

Visit http://www.dovepress.com/testimonials.php to read real quotes from published authors. 